\title{
The Automated Telescope of Novosibirsk State University
}

\author{
A. Nesterenko, M. Nikulin, D. Vyprentsev, A. Zaytsev \\ Novosibirsk State University, Pirogova 2, 630090, Novosibirsk, Russia \\ I. Nesterenko, V. Prosvetov, V. Tsukanov, A. Valishev \\ Budker Institite of Nuclear Physics, Lavrenteyva av.11, 630090, \\ Novosibirsk, Russia
}

\begin{abstract}
The current status of the $30 \mathrm{~cm}$ automated telescope project at Novosibirsk State University (NSU) observatory with Internet access is presented.
\end{abstract}

\section{Introduction}

The automated telescope facility was designed basically for photometric observations. The $30 \mathrm{~cm}$ reflector is intended to be used as a photometric monitor of variable stars and other objects at visible bands. A piggy-back $10 \mathrm{~cm}$ refractor will serve as a monitor of the solar surface. It is also planned to use the facility in the University education process. The NSU observatory location is $54^{\circ} 50^{\prime} 40^{\prime \prime}$ north latitude and $83^{\circ} 05^{\prime} 42^{\prime \prime}$ east longitude.

\section{The Optical Systems of the Telescopes and the UBVR Photometric System}

The facility will consist of two telescopes, a $30 \mathrm{~cm}$ Newtonian system (f/4.9) with a meniscus coma corrector and a $10 \mathrm{~cm}$ achromatic refractor (f/5.0). Calculations demonstrate, that the image is close to diffraction quality at the size of the CCD area sensors (ICX084AL and ICX075AL). For observations of the Sun it is necessary to use additional colour filters with transmission band at about $100 \mathrm{~nm}$. In this case the lens chromatism does not restrict the field of view in high quality images. For the sunlight reduction we use a full aperture filter with a transmission of about $1 / 10000$.

At the present time the $U B V R$ system is used for photometric measurements. Measured spectral response curves of the CCD camera with our filters have acceptable deviation from curves of the UBVR system.

\section{Hardware and Network Scheme}

The telescope control devices are presented in Fig. 1. The main $30 \mathrm{~cm}$ Newtonian and the piggy-back $10 \mathrm{~cm}$ achromatic refractor telescopes are placed inside a 4 


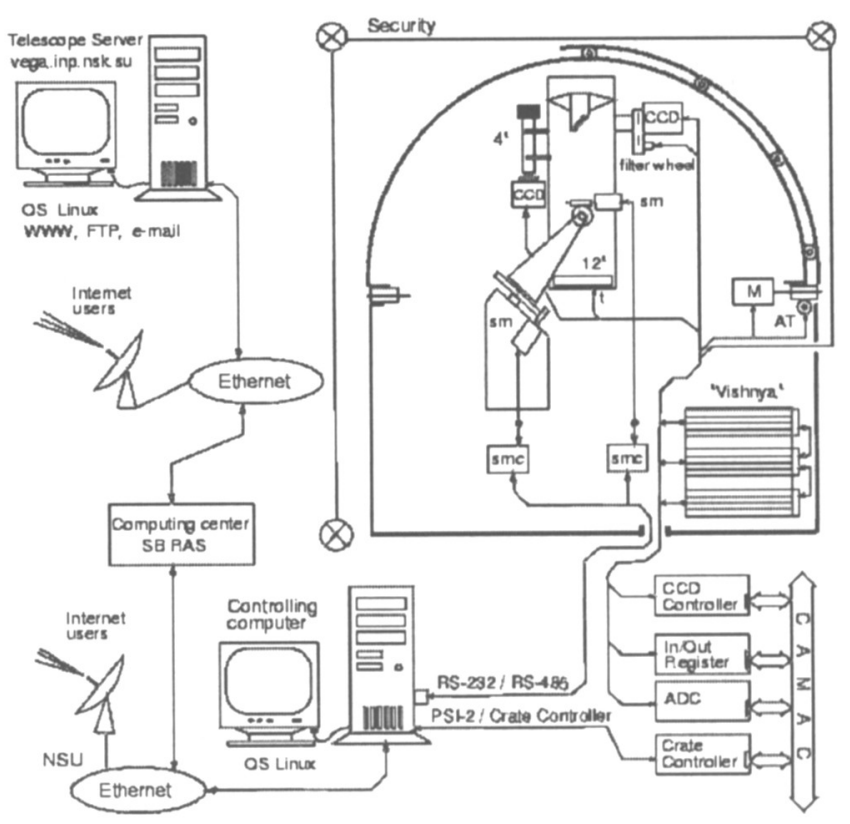

Figure 1. The hardware and network scheme

$m$ diameter dome. We use an equatorial fork mount equipped with two stepper motors to guide the telescope to the selected area in the sky. The motor control system consists of two independent stepper motor controllers SMC-485, which are connected to the standard computer RS-232 port via the RS-485 interface bus and the RS-232/RS- 485 converter. The RS- 485 interface allows us to connect up to 32 devices by a monochannel line with up to $1200 \mathrm{~m}$ length. The dome guiding system is based on two collector motors and potentiometric azimuth transmitter, which provides a rotation synchronism accuracy with the telescope of about $1^{\circ}$.

The control over all the automated telescope facility and the security system is performed by a computer powered by the GNU/Linux OS via the CAMAC and "Vishnya" (cherry in English) modules. Internet users can gain access to the telescopes after filling a registration form. This form and observation requests can be submitted by e-mail or filled interactively with a web browser.

\section{Conclusion}

The basic elements of the future automated telescope have already been built. The infrastructure to access this telescope through the Internet is basically formed. In the next year it is planned to complete the installation of the equipment and to initiate its commissioning.

The website of the NSU observatory is http://vega.inp.nsk.su/ 